\title{
Re-Thinking e-Learning Package Creation
}

\author{
Sun Teck Tan and Meng Wee Song
}

\begin{abstract}
Although video recording tools are abundant and easily available in the market, many of them are made for entertainment purposes and are far too complex for a non-trained user. The purpose of this paper is to explore the possibility of a dedicated application to record e-learning contents, with the specific tools in place and ease of use for users. Such tools may involve document viewers; whiteboards; webcam display; simple video editing tools; format encoder; automated uploads to learning management systems; etc. This paper will also shine a light on the current development of an application, future additions as well as results from the application. Insights on the issues pertaining to current video recording and editing tools will also be explored to give us a foundation on what is currently good and bad.
\end{abstract}

Index Terms-Content creation, e-learning application, video creation, video editing.

\section{INTRODUCTION}

With e-learning gaining popularity and being more widespread, it has become more imperative that we take a closer look at some issues that are currently plaguing e-learning. Many e-learning lessons are delivered through the means of recorded lessons, i.e. videos [1]. Viewing these videos is as easy as clicking and downloading yet the amount of work that goes into creating these videos are huge. Coupled with the fact that there are not many application dedicated to create e-learning packages, the job of those who creates such packages, mainly educator, only gets more complex. It was said that a quality e-learning content will take up to 200 man hours to produce and requires educators to keep up with technology [2], [3]. A recent survey also suggested information that technology is becoming more common in teaching and in classrooms [4], [5].

The lack of dedicated applications is what contributed mainly to these problems; there is not a single easy to use application that encompasses everything in it. If we wish to screen capture, we have applications like Camtasia; if we wish to view documents, we have Office Suites; if we wish to use a web cam, we would have to fire up another application and as such, applications make up a major role in e-learning. To the non tech-savvy educators, it would be a huge undertaking and a daunting task to learn and use so many different kinds of applications as technology, both hardware and software can be very complex. [6]

In this paper, a solution is proposed to address these issues, an application that will revolutionize the way e-learning

Manuscript received March 2, 2014; revised May 13, 2014.

The authors are with School of Computing, National University of Singapore, Singapore (e-mail: tanst@comp.nus.edu.sg, song_meng_wee@hotmail.com). packages are created. An all in one application that encompasses everything an educator would need to create their contents and being easy to use as it was designed for them from the ground up. In the next section, we will take a look at how our application differs from what is available in the market, a more in depth discussion about what is required from such an application as well as shining some light on the current developments.

\section{RE-Thinking E-LEARNING PACKAGE CREATOR}

\section{A. Issue with Current Applications}

Video creating or video editing software are of course in abundance on the market, however, the problem with them are that they are mainly made for entertainment purposes. Being entertainment tools, they feature many tools to add special effects, correct colors, animate and so on, but in e-learning context, most of such tools are unnecessary and actually add on to the complexity of the application. Most professionals that use such applications usually go through training and courses before they become competent enough to make full use of them and it is unfair to demand the same from educators, whose primary job was not to make videos.

Another important factor was the absent of several important teaching tools in these applications. Looking at many e-learning packages from various universities and academic institution such as OpenCourseWare from Massachusetts Institute of Technology (MIT) or e-learning courses from the National University of Singapore (NUS), one can easily notice that they feature some form of documents, such as PowerPoint slides or PDF documents. [7] Current e-learning packages have a trend of using screen capture program such as Camtasia to record, then using another program such as Microsoft PowerPoint to display those documents and perhaps another program to initiate an external camera feed. Not to mention that many conventional screen capture tools actually capture the whole monitor and thus shows many unwanted items such as desktop or other windows which may have served the recorder's script or notes. The point of this is that we can actually develop something that features everything an educator needs to teach his lessons in a video, is easy to use and does not even look complex to boot. This application will also feature a dedicated capture zone so that its user can actually make use of other spaces on his monitor for other purposes. Many of these features and their technical details will be further looked into in the next section.

Currently, Adobe Presenter from Adobe is one of the most prominent dedicated e-learning content creators available on the market and it serves as an add-on to Microsoft PowerPoint. It being an add-on to Microsoft PowerPoint would be its main problem, not only does that limit its 
document range to PowerPoint Slides only but for users without a document to feature, this application would also be unusable. The more crucial problem is its output format, being in flash format, it would deny users of iOS operating system, a mobile operating system found on iPhone and iPad, the ability to learn from their devices as the iOS operating system does not support this format. Another downside to being in flash format is the inability to share this content easily, many learning management systems (LMS) will support the uploading and streaming of video files very easily, as well as popular video sharing sites such as YouTube. In contrast to this, there is not really an easy way to share and host a flash format file.

\section{B. Tools and Features of an e-Learning Package Creator}

In this section we cover some of the most important tools pertaining to this application and why they are required. All of these tools are what makes this application so unique and different from what is currently available and truly defines a dedicated e-learning package creator.

\section{1) Document viewer}

As previously mentioned, most e-learning packages feature a set of document in their videos. To reduce the need for any external programs, integrating such document into the application itself is a must. Also, to make this application more versatile, this viewer must be able to support multiple document formats, especially more popular ones like PowerPoint and PDF. Due to this, various ports of this application might be needed to suit different operating systems because of office suites compatibility, for example, Microsoft Office Suites may only operate on Microsoft Windows and so on.

To make this more user friendly and to allow users to feel more at home, the layout of this viewer will be similar to that found in Microsoft PowerPoint. A main frame will feature the current slide/page of interest with an additional preview frame for subsequently slides/pages. Each of these frames will be resizable, moveable and removable to allow user more freedom in using this application and to allow them to create more dynamic and interesting packages.

\section{2) Whiteboard}

The whiteboard/blackboard has always been a staple tool in an educator's arsenal to teach and is one of the most effective means of conveying or elaborating on ideas that are otherwise dormant in their minds. It is a useful tool for writing down equation, explanation or to even draw diagrams that may help learners understand better. As such, any application dedicated to e-learning should have a whiteboard in built and this application itself is no exception. The whiteboard by itself is a simple tool that allows its users the ability to write anything on the application itself with many different pen sizes and ink colors. A highlighting capability was also added to allow user another way to emphasize on certain items/sentences on the video itself. The idea behind each and every tool was to allow them to synergize well with each other, for example, with the whiteboard and document viewer, an educator can highlight certain parts of the document or write equations beside to explain further in details.

\section{3) External camera support}

In a traditional classroom setup, it is easy to show experiments or other items to students to ignite their interests and intrigue them, but in e-learning, this simple task can be quite hard to accomplish. Using an external camera or an in-built laptop webcam does allow the teacher a mean to show any experiments, but it always require additional programs to activate them. Another method is to record the experiment prior to recording the lesson but this would require more work, synchronization and some sort of video editing software. Therefore, it is imperative that this application has a way for its user to activate any external cameras that they may have connected to their computer. Multiple camera support can also be added to allow users more freedom on how they wish to create their packages.

\section{4) Video player}

Why would a video recorder application require a video player as one of its tool? Well, there are times where a teacher may wish to show relevant videos from another source to their students and these videos may resides on the computer running the application itself or some online video sharing site like YouTube. For videos residing on hard disk, it is easy to play and display them in the application even with functions like resizing. Many code libraries have already supported such feature and integrating them will also not be too much of an issue.

The main problem however, comes from streaming video from streaming sites on the application itself. To reduce the application's complexity, we opt not to let the user download the video first then play it but rather to directly stream the video on the application. YouTube will be the main streaming site supported by this application due to its huge user base and large library of contents which even include e-learning contents such as those from Kahn Academy or MIT OpenCourseWare. Although Google provides their own API for integrating YouTube into any application, research and work still has to be done before decision are made on whether it would be better off creating a video streamer from scratch. The main comparison here is on whether YouTube would be the only streaming source or would other streaming source be also incorporated into this application in future and also the versatility of the Google API to work with other video streaming sites.

\section{5) Dedicated capture zone}

Although there are tons of screen capture applications available in the market, many of them actually captures the entire screen that the user sees himself. Running a full screen program would of course be ideal in this sort of scenario but there also times where a user decides to use other parts of the screen for other purposes such as reading his script. What we aim to achieve is to create a dedicated capture zone where all the screen capturing are done, this zone can vary size to suit the video resolution that the user wishes to use. Outside of this zone, we can place the control panel for the various tools previously mentioned and even have a dedicated area for note taking or script reading. Because of such a setup, it also allows the developer more freedom in deciding where to place certain tools when creating them, if the control for the tool would be more beneficial to be seen in the video, it can 
easily be moved into the recording frame and vice versa.

\section{6) Editing}

No matter how perfect the recording feature, no video creator is complete without an editing tool, even if it is just a simple one. One of the main issues with current video editing tools is the fact that they are too complex and have many redundant features in the context of e-learning. Video editing in e-learning is mainly used to correct minor errors made or to add content that the recorded have previously missed out. Due to this, the only feature that we really need for editing are cutting and joining of videos and not features like color correction, tilt adjustment and so on. Both cutting and joining tools will allow user to select the time, down to the seconds, on which they wish to remove or add in new videos.

The editing tool will work with most conventional video file; however, when paired up with videos that are recorded using this application, a "by slide" feature will be enabled. This feature will allow the removing or addition of video via slides instead of timestamp. For example, clicking on cut followed by clicking on slide 3 will remove the entire section that features slide 3 , the same goes for joining, where the added video will be added right after slide 3 . This feature will allow educators who make adjustments to their original document an easy way to make the same adjustment to their videos.

\section{7) Encoding}

One of the major issues with Adobe Presenter is its output format not being compatible with mobile devices or streaming site. Due to this, it was decided for the .MP4 format to be the output format for this application. This format is supported by Android and iOS devices as well as various popular video streaming sites which allow the output to be placed and viewed anywhere easily. However, there are some technical factors inside a MP4 format such as audio streams, bit rate or frames per second that also has to be considered in order to make it truly universal. The small file size of MP4 also makes sharing and uploading much easier and time-saving. Of course by limiting the output to that of a video format, we are actually making it much less interactive as compared to flash format, albeit much more convenient. Users would not be able to set quizzes or questions in the video itself but we believe that it is a fair trade off for convenience and with the possibility of the videos being hosted on a LMS instead of a normal streaming site, we can actually make use of the LMS to generate the quizzes instead.

\section{Current Developments}

Earlier, we briefly touched on how the document viewer frames are resizable, moveable and removable. These frames are actually the main container for most of the tools featured in this application such as webcam or video players. They allow developers an easy way to add additional tools into the application without worry of modifying any of the core codes Tools added this way will also feature the same function of being resizable, moveable and removable without the developer doing any extra work.

Any picture shown here is at the point of the writing and may differ from what the application actually looks like now as development is on-going.

\section{1) Recording window}

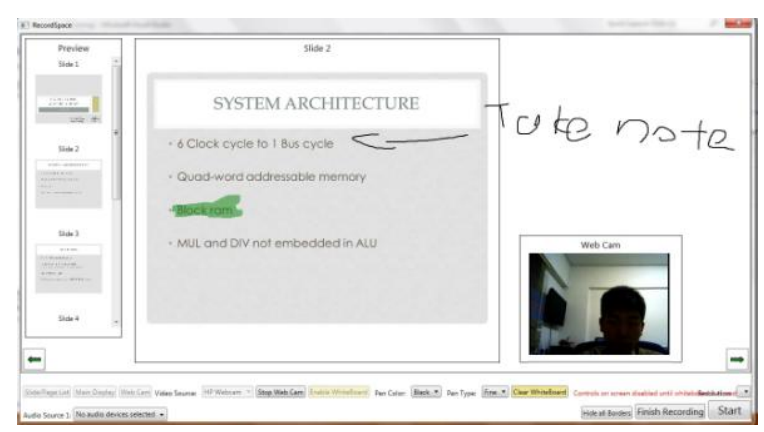

Fig. 1. Prototype of recording window.

A picture means a thousand and through Fig.1, one can clearly understand how every single tool is able to come together for a single e-learning video. Tools that are featured above include:

- Document viewer (Both main frame and preview frame)

- External camera support

- Whiteboard

- Dedicated capture zone

The capture zone is actually the upper rectangle and does not include the array of buttons that are being displayed on the bottom bar, which is actually the control panel. For the document viewer itself, we tried to mimic the familiar PowerPoint layout as much as possible to make user feel more comfortable but do note that they are actually removable via the control panel, resizable and moveable. As you can see, the whiteboard tool synergizes really well with the document viewer, being able to highlight important texts and make additional notes on the side will allow the teacher to focus attention on the area he deems necessary.

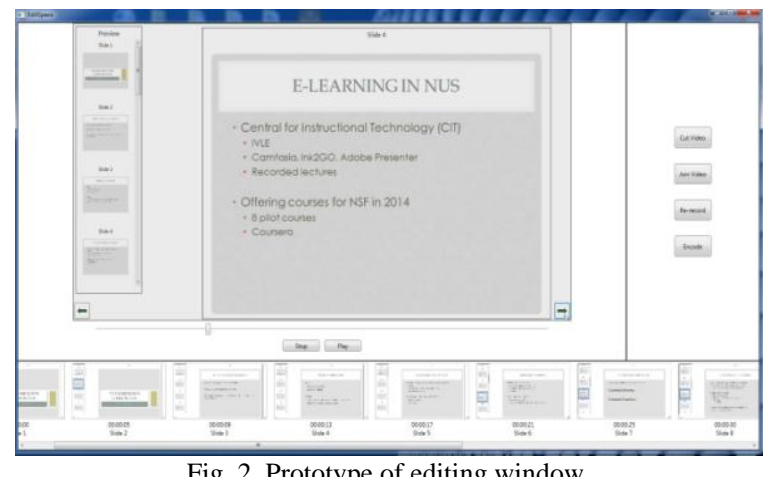

The webcam frame is also resizable but the maximum size of it is however limited by the maximum resolution of the camera used. With this frame, experiments or any external item may also be shown in the final video itself to allow for a more interactive experience. The ability to hide any of these frames at any time allows for the educator to shift the focus of the students easily to the important parts instead of being distracted. It also allows for the entire canvas to be used as a simple whiteboard or a full size webcam viewer, the possibilities are endless with such a system. And yet, this is just the initial development of this application, with future addition of other interesting tools and features, one can only imagine how this system will eventually looks like.

\section{2) Editing window}

Fig. 2 showcases the editing window of the current 
development with a video recorded via the application itself. Because the video was recorded via the same application, the editor was able to detect which slide was featured at which timestamp as seen in the preview pane on the bottom. This editor is able to work with any MP4 file but the slide number will not be shown or used if:

- The video was not recorded by the application itself

- The accompanying binary file was removed

- The video does not feature a document

The binary file is a file that is generated from the recording tool when a video that features a document is recorded. The application will then pair each slide/page transition to the corresponding timestamp and record them onto the file. Currently this file is an external file with the same filename as per its accompanying video file but future plans will be to embed this information into the video file itself to prevent accidental deletion. Next, we will take a look the right side of the editing window which is actually the control panel with features like:

- Cut Video - which remove a section of the video base on timestamps

- Join Video - which adds another clip to the current video base on timestamps

- Re-record - allows user to re-record a clip using this application and join it base on timestamps

- Encode - Publish the final video in MP4 format

The concept that revolves around this window is based on simplicity and user friendliness, with minimal yet enough function to suit every users need. The entire page speaks for itself without a need for any manual or guidance on how to use each function available.

\section{3) Output}

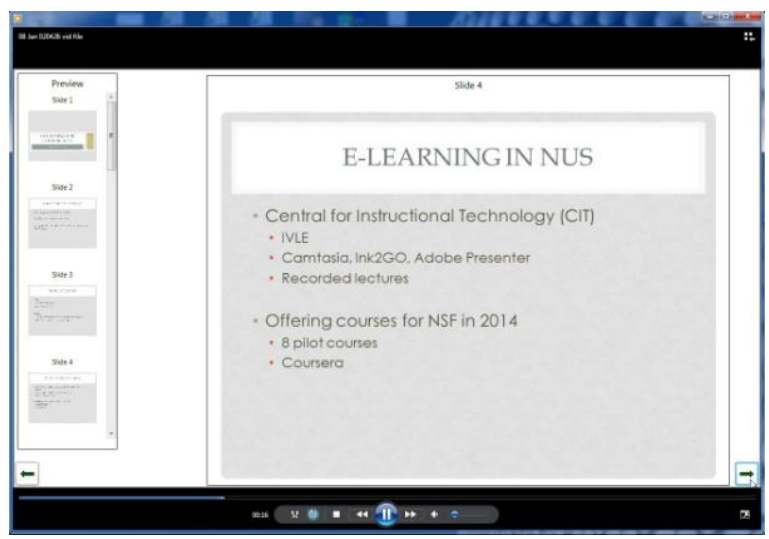

Fig. 3. Prototype of editing window.

Fig. 3 shows Window Media Player playing the final output video from the application itself, as can be seen from the picture, the control panel or tool bar are not visible as they did not belong inside the capture zone. The result is a clean and clutter free video that have offers no distracting elements to the student so that they may better focus their attention. What you see here is just the icing on the cake; plans are still being made on how to further develop this application and will be discussed on the next section.

\section{FUTURE DEVELOPMENTS}

Being a dedicated application for e-learning, one of the feature that could be incorporated into this is the ability to pair up with a LMS. This could range from simple features like automatic uploading of finished videos onto the LMS to more complicated feature like quiz creation. This allows for a more complete e-learning package based upon one creation tool and one delivery tool and will greater reduce the man hours required to create any content.

Another planned development is a port of the current application to other operating systems. The current application is based on windows and works with Microsoft Office suites. The major issue with this is the reliance of certain office suites to a certain operating systems, for example, Microsoft Office with Windows. Due to this, if development were to occur for Apple OS, the document viewer and linker would have to be re-developed to suit the office suites that Apple OS is using. For office suites that have no operating systems requirements such as OpenOffice, the document viewer and linker would just have to be updated with the API of the corresponding suites.

We mentioned earlier that we plans to incorporate information pertaining to slides and timestamp into the video file itself. This information, other than being used for editing, could potentially be used for video playback as well, with a dedicated video player; we could allow learners to navigate to and fro slides/pages easily. However, this file would also need to be playable on a conventional video player as to limit the output video to only one single player.

\section{CONCLUSION}

This system clearly give its user an edge when it coming to creating videos, by giving them a dedicated space and all the tools they need to better transfer their ideas and teachings, the video created also looked better, are more interesting and cleaner compared to conventional applications. The idea was to develop a system that could evolve and keep up with the times, and also being useful and user friendly at the same time. Not only for the user but also for the developer, the system will be easily updatable with any newer technology that may come out in the future. Although many of that is achieved and a basic system is already out, much more work still has to done to perfect this system so that it may, one day revolutionize the entire e-learning scene.

\section{REFERENCES}

[1] Evaluation of evidence-based practices in online learning, SW Washington, DC, 2009 PP. 37-40.

[2] M. Gleeson. (August 2013). Why e-learning is so hard. [Online] Available: http://mgleeson.edublogs.org/2012/08/08/elearningishard/

[3] C. Tucker. (March 2014). Time Estimates for e-learning Development Experiencing e-learning. [Online]. Available: http://christytucker.wordpress.com/2014/03/18/time-estimates-for-e-le arning-development/

[4] P. Brogan, Using the Web to Train Knowledge Workers, The Imperative for the New Millennium, San Francisco, CA: Macromedia, 2007, pp. 13-15.

[5] PBS Learning Media. (February 2013). Teachers embrace digital resources to propel learning. Public broadcasting services. [Online]. Available:

http://www.pbs.org/about/news/archive/2013/teacher-tech-survey/

[6] P. Weaver, Avoiding e-learning failure, Bridgeville, PA: DDI, Aug 2002, pp. 4-5.

[7] D. Wilson, T. Callaghan, and S. Honore, E-Learning, the Future of Learning, Cirencester, U.K.: Elearnity, Jan 2000, pp. 24-27. 


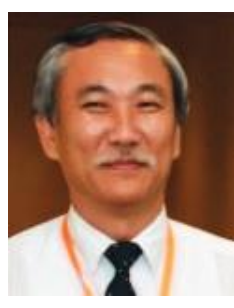

Tan Sun Teck is an associate professor with the School of Computing (SoC) in the National University of Singapore (NUS). He graduated from the old Nanyang University in Singapore with a BSc in 1980 and the University of Essex, UK with MSc and $\mathrm{PhD}$ in 1984 and 1989 respectively. He has been lecturing in NUS since 1989, first with the Department of Information Systems and Computer Science, and then in SoC when the Department became a fully-fledged faculty called SoC. Dr Tan's research interests includes Chinese Computing and Computer Aided instruction.
Song Meng Wee was born in Singapore in 1989. He currently holds a diploma in computer engineering from Temasek Polytechnic, Singapore (2006).

He is currently an undergraduate at the National University of Singapore in his 4th year working towards his bachelor of engineering, Computer Engineering and has worked as a software engineer prior to this. 